\title{
Hacia una taxonomía de los equipos y sistemas técnicos empleados en la interpretación simultánea
}

\section{(Towards a taxonomy of technical equipment used for Simultaneous Interpreting)}

\author{
Aurora Ruiz MEZCuA \\ Universidad de Córdoba
}

Fecha de recepción: 30 de mayo de 2011

Fecha de aceptación: 11 de julio de 2011

Resumen: El mundo de la interpretación ha cambiado radicalmente desde la aparición de las tecnologías que han propiciado el surgimiento de la interpretación simultánea. Esta es una profesión joven que se basa en el uso de material tecnológico. A pesar de tratarse de un modo de interpretación que prácticamente depende de la utilización de un equipo técnico que habilite la escucha y la producción oral "al mismo tiempo", los trabajos científicos que se le han dedicado son escasos. El propósito de este artículo reside en analizar la tipología de los equipos de interpretación que pueden encontrarse en el mercado profesional en la actualidad.

Palabras clave: Traducción. Interpretación. Equipo electrónico. Sistema de interpretación.

Abstract: The world of Interpretation has greatly evolved since the emerge of the technology which gave birth to simultaneous interpreting. SI is a young profession based on the use of a technical system. Despite the fact that this popular mode of interpreting relies heavily upon an electronic equipment that enables listening and speaking "at the same time", there have notably been few scientific studies on the use of this equipment in the SI field. The aim of this article is to analyse the types of SI equipment than can be found in today's professional market.

Key words: Translation. Interpreting. Electronic equipment. Interpreting system

\section{INTRODUCCIÓN}

"Un vrai aquarium" (Keiser, W., 2004: 32)

"Un auténtico acuario", estas son las palabras textuales de MarieFrance Skuncke, intérprete en los juicios de Núremberg ${ }^{1}$ y profesora de interpretación simultánea en la Universidad de Ginebra, al recordar el equipo donde trabajó por primera vez. Han pasado bastantes años desde entonces y, sin embargo, muchos son los profesionales que actualmente se

\footnotetext{
${ }^{1}$ A lo largo de este trabajo utilizaré la grafía Núremberg, pues es la adaptación española que el Diccionario de la Real Academia propone para el topónimo alemán Nürnberg.
} 
sienten identificados con esta angustiosa sensación cuando llegan al lugar donde van a interpretar. Estas cabinas, popularizadas fuera del gremio gracias a la película estadounidense titulada $\mathrm{La}$ intérprete ${ }^{2}$ forman parte de un conjunto de instalaciones y dispositivos que suponen el resultado de experimentos y mejoras tecnológicas que han servido para introducir una nueva forma de interpretación: la simultánea.

Sin embargo, este equipo ha sufrido menos metamorfosis de las que podríamos imaginar, habida cuenta de la evolución que en las últimas décadas han experimentado otros elementos tecnológicos. Ahora bien, no todos los sistemas de interpretación son idénticos. Si bien es cierto que podemos encontrar algunos dispositivos comunes a todas las clases de equipos, no debemos obviar que también presentan diferencias. Por ejemplo, no se utilizan las mismas instalaciones en un congreso del mercado local $^{3}$ de una de las capitales de provincia españolas donde se celebren unas jornadas de tres días sobre un tema peregrino, que en una sala de conferencias de la Organización de Naciones Unidas.

\section{QUÉ ES UN EQUIPO DE INTERPRETACIÓN SIMULTÁNEA}

El Diccionario de la Real Academia de la Lengua Española define equipo en su quinta acepción como la "Colección de utensilios, instrumentos y aparatos especiales para un fin determinado"A. En este caso, ese fin determinado sería el hecho de que pueda realizarse la interpretación simultánea ${ }^{5}$. Dado que esta definición es poco precisa, prefiero la propuesta por Inés Drallny: "Se entiende por equipo electrónico las consolas y equipamiento diseñado y construido especialmente para las necesidades de la interpretación" (2000: 29). Esta, a su vez, aparece definida en el diccionario académico en una acepción dentro del adjetivo "simultáneo" y

\footnotetext{
${ }^{2}$ Su título original es The Interpreter y se trata de un thriller dirigido por Sydney Pollack y protagonizado por Nicole Kidman y Sean Penn. El argumento relata las aventuras de una intérprete que de forma accidental escucha, a través de unos cascos de interpretación y desde una cabina, una operación para atentar contra el presidente de la "República Democrática de Matobo". Resulta especialmente curioso el subtítulo de la película, que supuestamente escrito en "kú" reza "Angota ho ne njumata", que en español significaría "la verdad no necesita traducción".

${ }^{3} \mathrm{Al}$ que algunos investigadores también se refieren como "mercado libre" (Martin, A., 2000: 171).

${ }_{5}^{4}$ En la vigésima segunda edición aparecen seis acepciones diferentes.

${ }^{5}$ En realidad, además de la IS, existen otras variedades. En el estudio de la interpretación aparecen discrepancias terminológicas que en ocasiones afectan a la propia descripción de las modalidades (que suelen definirse según el contexto en el que se realicen o la técnica que se emplee). No obstante, normalmente, se suele distinguir entre cuatro tipos de interpretación: "interpretación simultánea", "interpretación consecutiva", "interpretación de enlace" y "traducción a la vista" (Bordons, B. y Jiménez, A., 1996: 227), por un lado, y dos contextos: interpretación de conferencias e interpretación social.
} 
con el nombre de "traducción simultánea", aunque dentro del mundo de la traductología suele distinguirse entre la traducción y la interpretación. Esta última suele hacer referencia a las ideas que se expresan oralmente de una lengua a otra, por contraposición a aquellas que se expresan por escrito -que sería la traducción- (Munday, 2001: 4).

De este modo, entenderemos "interpretación" como "la transmisión de mensajes orales entre dos lenguas (...) transfiriendo todos los componentes para que dos personas que no comparten una misma lengua puedan interactuar sin que el idioma sea una barrera" (García Beyaert y Serrano Pons, 2009: 56). Se trata, por lo tanto, de una definición general en la que se engloban todos los subtipos de interpretación de lenguas orales (no ocurriría lo mismo con la lengua de signos, que quedaría fuera del enunciado, pues la comunicación se lleva a cabo por un canal diferente ${ }^{6}$ ). Quizás esta explicación podría mejorarse si en lugar de utilizar la frase "para que dos personas que no comparten una misma lengua", se incluyera "varias personas", pues en muchas ocasiones, el número de individuos que necesitan la interpretación es superior a dos. En ese sentido la definición que propone Keiser me parece muy clarificadora, pues describe el proceso de la interpretación simultánea a través del uso del equipo electrónico y las necesidades del intérprete en el contexto de la interpretación de conferencias:

The interpreter works in special booths and listens through earphones to the speaker in the conference room, watching at the same time what is going on in the meeting room (projection, etc.) through the booth window. As the speaker's statement proceeds, it is translated simultaneously into the other language or languages of the conference and fed back through booth microphones to earphones in the meeting room. Delegates can listen in whichever language they wish by switching their earphones to the appropriate channel. (Keiser, 1978: 15).

Una vez aclarados estos conceptos, cabe destacar que este trabajo se centrará en el estudio y clasificación de los instrumentos y dispositivos especiales que se necesitan para efectuar la interpretación simultánea en la que se utiliza equipo electrónico.

\footnotetext{
${ }^{6}$ La lengua de signos es "el modo de expresión utilizado por las personas carentes de audición. Se trata de un sistema de audición distinto que el oral en cuanto al modo de producción y percepción del lenguaje, posee un código específico que se articula con las manos y se percibe visualmente (...) la lengua de signos no es universal, no solamente es icónica, posee propiedades de creatividad y recursividad, así como unidades contrastivas" (Jorge Figueroa 1998: 146). La lengua de signos, por lo tanto, queda fuera de este estudio, ya que no se necesita equipo técnico.
} 


\section{UN POCO DE HISTORIA}

El equipo de interpretación simultánea fue creado por la empresa IBM e introducido en la Liga de Naciones, en concreto en la Organización Internacional del Trabajo en 1927, gracias a la colaboración de Filene, hombre de negocios que había asistido a las reuniones de la organización y había atestiguado los problemas derivados de la interpretación consecutiva; Finlay, ingeniero de sonido, y Watson, director de IBM. La posibilidad de escuchar un discurso y su traducción casi al mismo tiempo a través de un sistema de auriculares, micrófonos y cables suponía, en aquella época, un avance prodigioso ${ }^{7}$ que propició el nacimiento de una nueva modalidad que poco a poco se convertiría en la técnica más común en las reuniones internacionales multilingües: la interpretación simultánea.

De este modo, la interpretación simultánea como la conocemos actualmente se viene practicando desde que su equipo se puso en funcionamiento en 1927 con motivo de la Conferencia de la Organización Internacional del Trabajo. Con el paso del tiempo, se realizaron mejoras tecnológicas significativas que hicieron posible que pudiera utilizarse en otras situaciones. Algunas de las más destacadas son, por ejemplo, las subsecuentes asambleas de la OIT o el XV Congreso de Psicología de Leningrado, donde la sesión inaugural (pronunciada por el profesor Pavlov) fue interpretada del ruso al francés, alemán e inglés.

Con la llegada de la II Guerra Mundial y los problemas de la Liga de Naciones, la evolución e introducción del equipo en nuevos eventos se vio paralizada, o adormecida, para luego resurgir con más interés en uno de los juicios más conocidos de la historia: Núremberg. Los Aliados establecieron un Tribunal de Justicia para condenar los actos nazis que se habían llevado a cabo en Europa y necesitaban dar a conocer los resultados lo antes posible e involucrar al máximo a los medios de comunicación para difundir el evento. La solución era instalar el equipo de interpretación simultánea (llamado hushaphone Filene-Finlay) en las salas correspondientes para poder utilizar la nueva técnica y realizar intercambios lingüísticos en los cuatro idiomas requeridos a tiempo real. Sólo dos intérpretes de los que trabajaron en este juicio tenían experiencia previa trabajando en simultánea, el resto recibieron una escasa formación días antes de la primera sesión ${ }^{8}$. Las condiciones no fueron las más adecuadas, sin embargo, con algunas de las mejoras que se pudieron realizar al equipo que IBM había suministrado y

\footnotetext{
${ }^{7}$ Aunque para algunos intérpretes se trataba de una desvirtuación de su profesión, pues en aquella época se practicaba sobre todo la modalidad consecutiva, que se consideraba más prestigiosa.

${ }^{8}$ Los investigadores Francesca Gaiba y Jesús Baigorri, entre otros, han abordado con detalle el tema de la formación de los intérpretes de Núremberg (Gaiba, 1998: 49 y Baigorri, 2004: 288).
} 
la presencia de los técnicos (pertenecientes en parte al ejército estadounidense, al igual que algunos componentes del sistema), la interpretación simultánea fue un éxito y demostró que sería el futuro de la profesión.

De esta forma, gracias a este gran logro de Núremberg, la Organización de Naciones Unidas se interesó por la nueva técnica y su innovador sistema. Primero lo instauró de forma experimental y luego terminó prácticamente sustituyendo a la consecutiva, y este fue el paso definitivo para que el equipo se instalara en numerosos tribunales y organizaciones internacionales, salas de conferencias y palacios de congresos.

Sin embargo, cabe apuntar que, aunque técnicamente se ha avanzado mucho, los dispositivos que conforman el equipo siguen siendo básicamente los mismos. Esto significa que las nuevas tecnologías han aportado una mejora en la calidad del sistema de sonido, aislamiento, escucha, adición de ordenadores, etc., pero el equipo no ha experimentado un verdadero cambio o evolución, y aún hoy pocas son las personas conscientes de la necesidad de trabajar utilizando un sistema de calidad.

Uno de los elementos primordiales que el intérprete necesita para poder realizar su trabajo es escuchar al orador. Para ello, las condiciones de sonido deben ser óptimas, cosa que no ocurre en la mayoría de las ocasiones debido a la baja calidad del equipo técnico, que con demasiada frecuencia es antiguo y no puede satisfacer las demandas de sus usuarios. Resulta inaceptable que el intérprete tenga que dedicar una gran parte de su atención y energía a intentar escuchar con claridad lo que el orador dice, en vez de centrarse en la comprensión y reformulación del mensaje. Los trabajos más significativos que se han elaborado a este respecto hacen referencia a los requisitos establecidos por las Normas Internacionales (principalmente por tres de ellas: ISO 2603, ISO 4043 y CEI/IEC 60914), secundadas por las principales asociaciones de interpretación, como AlIC o AIT, cuya creación es relativamente reciente.

\section{EL EQUIPO ACTUAL DE IS}

Antes de comenzar con la descripción de los equipos que se emplean en la actualidad para efectuar la IS, considero imprescindible aclarar que no todos los equipos actuales cuentan con las mismas características. Por ejemplo, en los organismos internacionales y otros lugares de prestigio donde se realiza la interpretación simultánea con frecuencia, el equipo técnico es más sofisticado y dispone de un mayor número de funciones o incluso de dispositivos secundarios, mientras que en las salas de conferencias donde la interpretación simultánea es una técnica que se utiliza en contadas ocasiones, la instalación es más sencilla. 
Hay veces en las que ni siquiera se dispone de un equipo en el lugar donde se quiere celebrar el acto para el que se necesita la interpretación. En esos casos, los organizadores del evento se ponen en contacto con empresas que alquilan equipos portátiles. Así, la primera clasificación que voy a realizar es la relativa a la instalación de los equipos y distinguiré entre portátiles y fijos. No obstante, esta categorización resulta insuficiente, pues existen varios subtipos, cuyas características dependen de la función para la que se han diseñado. Así, por ejemplo, podemos encontrar equipos portátiles que se utilizan en las mismas situaciones que los fijos y, por lo tanto, deben disponer de los mismos elementos que éstos. Por otro lado, también podemos ver equipos ideados para utilizarse en otro tipo de situaciones o para otros fines. Por ejemplo, equipos móviles diseñados para que tanto los asistentes como el intérprete y el orador puedan desplazarse.

Parece conveniente, por tanto, realizar una segunda clasificación basada en los contextos de utilización de los equipos, pues de estos depende su diseño. Así, distinguiré entre equipos que se utilizan en el ámbito de la interpretación de conferencias y equipos que se utilizan en otros contextos interpretativos. Llamaré a los primeros equipos convencionales y a los segundos otros equipos de interpretación ${ }^{9}$. Estos últimos dispositivos son menos frecuentes, pero no por ello menos importantes. En realidad, su uso resulta más sencillo, ya que no es necesario disponer de una cabina de interpretación. Al mismo tiempo, su calidad suele ser inferior, en el sentido de que no existe insonorización, no se puede trabajar con ellos durante un gran periodo de tiempo, etc. En las páginas que siguen explicaré con detalle cada una de las tipologías, comenzando con un esquema con la clasificación propuesta:

\footnotetext{
9 Algunas empresas de fabricación y alquiler de equipos denominan a los primeros equipamientos técnicos y a los segundos equipos portátiles para la interpretación simultánea, "portable translation facility", "portable interpreting equipment", etc. Esta terminología no me parece apropiada, pues los equipos portátiles que se utilizan en el ámbito de la interpretación de conferencias cuentan con los mismos componentes que los fijos, a pesar de que su instalación sea temporal y, sin embargo, son muy diferentes de los que se utilizan en otros contextos, como veremos a continuación.
} 


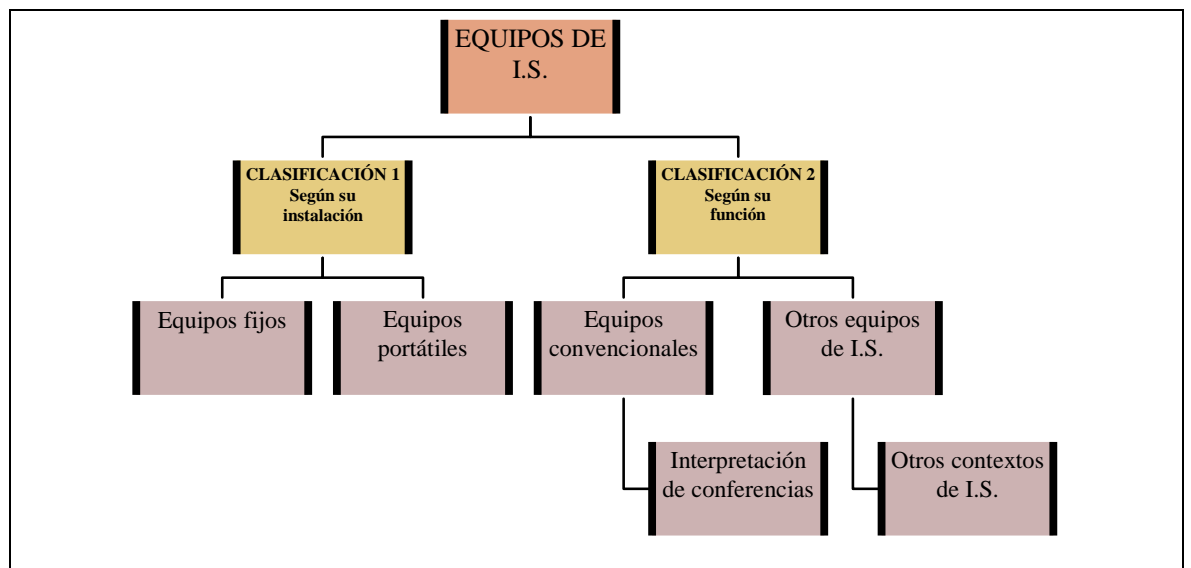

Clasificación de los tipos de equipos actuales

\section{a) Equipos convencionales}

Independientemente de su naturaleza y tipología, en la actualidad podemos considerar como equipo de interpretación simultánea convencional: la cabina, el sistema de sonido y la consola. Estos son los tres elementos básicos que no deben faltar ni fallar para que se pueda realizar un trabajo de interpretación adecuado en los contextos de conferencias. De aquí en adelante me referiré a estos elementos como componentes principales. Con esta expresión los diferenciaré del resto, que denominaré componentes secundarios (sistema de grabación, unidades de voto $^{10}$, etc.).

A continuación, incluyo un cuadro aclaratorio en el que se muestran los componentes principales de los equipos convencionales de interpretación simultánea.

\footnotetext{
${ }^{10}$ En ocasiones éstos no son realmente componentes del sistema de interpretación, sino accesorios diseñados con otras finalidades.
} 
Componentes principales:

* una consola para cada intérprete

* una cabina para cada equipo de intérpretes ${ }^{11}$

* el sistema de sonido: auriculares individuales y micrófono individual

Componentes principales del equipo de IS

\section{a.1.) La consola de interpretación}

Uno de los elementos principales del equipo es el cuadro de control, pupitre de control del intérprete o consola de interpretación ${ }^{12}$. La importancia de este dispositivo radica en que contiene todos los mandos necesarios para la escucha (recepción) y el habla (producción) ${ }^{13}$. Se trata de un módulo formado básicamente por un panel de control, un micrófono (que puede ser extraíble o fijo) ${ }^{14}$ y unos auriculares (que están conectados al módulo). Las consolas más modernas son individuales y tienen un display alfanumérico.

El panel de control está formado por varios selectores que se utilizan para diversos fines. En algunas ocasiones estos selectores son botones que pueden pulsarse para activarlos, en otras son ruedas que se pueden girar y, en otras, palancas con varias posiciones. La forma o apariencia de estos controles dependerá de los modelos y las diferentes empresas que se hayan encargado de su diseño y fabricación. Dada la distinta naturaleza de sus usos, suelen ser fáciles de distinguir entre sí. Lo más habitual es que aparezcan organizados por grupos y que, según esta clasificación, se sitúen en las distintas partes del cuadro de control: zona de escucha, zona de

\footnotetext{
${ }^{11}$ Algunas organizaciones recomiendan el uso de una cabina por idioma activo. Esto sucede cuando el equipo de intérpretes trabaja en lo que dentro del ámbito de la interpretación se conoce como "cabina pura", es decir, el intérprete escucha la lengua origen pero sólo utiliza una lengua meta (sin retour). Cuando se hace "cabina mixta", el intérprete trabaja en directa e inversa, con dos lenguas que pueden ser origen y meta dependiendo de las circunstancias; por lo tanto, para éste último caso deberíamos referirnos a "combinación lingüística" y no a "idioma activo", puesto que serían al menos dos las lenguas que el intérprete emplearía activamente dentro de la cabina de interpretación.

${ }^{12}$ Las diferentes denominaciones se deben a la integración o no en la estructura de la cabina. Si está montada sobre una base independiente, se denomina pupitre (forma habitual para un equipo portátil), si no, cuadro de control. Según las fuentes consultadas, el término que engloba los distintos tipos es consola, de ahí que haya titulado el punto de esta manera.

${ }^{13}$ Lo cierto es que se podría considerar como parte del sistema de sonido, pero debido a su mayor complejidad y número de funciones en comparación con el resto de componentes, me parece mejor estudiarlo como un elemento aparte.

${ }^{14}$ Cada intérprete debe trabajar con un micrófono, que puede estar sobre una base individual, incorporado a una consola o bien montado con los auriculares formando una unidad.
} 
micrófono, zona de control y zona de selección de canal. Así, por ejemplo, los controles de "escucha" y de "habla" siempre están separados.

Tipos de controles:

* Selector del canal de entrada y selector del canal de salida.

* Control del volumen y control de tonalidad.

* Control de apagado y encendido (on/off) del micrófono.

* Silenciador (mute).

* Selector para el relé de escucha (relay).

* Selector para volumen de micrófono.

* Altavoz de seguimiento.

\section{a.2.) La cabina}

Podemos definir este componente como el habitáculo desde el cual el intérprete realiza la labor de traducción. Según las Normas Internacionales $^{15}$, podemos distinguir tres tipos de cabinas diferentes ${ }^{16}$ :

* Cabina con equipo fijo: cabina prevista para la interpretación simultánea, dotada de un equipo de interpretación instalado de forma permanente (cabinas de obra). Suelen encontrarse en palacios de congresos, grandes salas de reuniones, etc.

* Cabina con equipo portátil: cabina prevista para la interpretación simultánea, pero no dotada de equipo de interpretación fijo (éste suele alquilarse para la ocasión). Se puede encontrar sobre todo en hoteles, centros de formación, empresas privadas, cámaras de comercio, sedes de asociaciones, etc.

* Cabina semipermanente: cabina que no se halla integrada en la estructura del edificio o que está concebida para ser desplazada dentro de éste. Son menos frecuentes que las dos anteriores.

Las cabinas se utilizan principalmente por tres motivos: el primero de ellos es que se necesita una separación acústica entre los diferentes idiomas que se hablan simultáneamente (tanto de los intérpretes como del orador) y un aislamiento del ruido que se produce en la sala. El segundo es que, al mismo tiempo, debe haber una comunicación entre el intérprete, el orador y el público, así como visibilidad de la sala; y el tercero que el intérprete necesita disponer de determinado material y de unas condiciones

\footnotetext{
${ }^{15}$ En concreto la norma ISO 2306.

${ }^{16}$ Mario León explica de forma despectiva en qué consiste lo que él llama "medias cabinas", es decir, un cuadro acristalado que se coloca encima de una mesa para que el intérprete introduzca en él la cabeza. Este autor considera que dicho elemento es puramente decorativo y "debería desaparecer del mapa congresual por su inoperancia” (León, 2000: 252). Personalmente, no he tenido la oportunidad de ver ese tipo de cabinas, pero por la descripción dada, comparto la opinión de León.
} 
de trabajo cómodas que le permitan mantener el intenso esfuerzo de concentración que se requiere.

Por las dos primeras razones, las cabinas están formadas por cuatro paredes, un techo, una vidriera ${ }^{17}$ y una puerta (que ha de ser silenciosa). Estos siete elementos deben asegurar el aislamiento acústico. Con el fin de evitar fenómenos de resonancia, las tres dimensiones de la cabina deben tener medidas diferentes y, para eliminar ondas estacionarias, las dos paredes laterales no deben ser exactamente paralelas.

Para la fabricación de las vidrieras, a través de las cuales el intérprete puede ver la sala y al orador, y gracias al nivel tecnológico que se ha alcanzado en la actualidad en materia de elaboración de vidrio, se utiliza un solo cristal laminado del grosor conveniente. Para que no se produzcan reflejos, las cabinas deben contar con una iluminación de trabajo proporcionada por focos en el techo. Lo más frecuente es que estos focos sean halógenos. Asimismo, y en consonancia con el tercero de los motivos por los que se utilizan cabinas, se debe tener en cuenta que éste es el lugar donde se albergan todos los dispositivos que el intérprete maneja, es decir, la consola, el micrófono, los auriculares y, en algunas ocasiones, los monitores; además del material que el propio intérprete puede llevar a la conferencia, como diccionarios, glosarios o un ordenador portátil, entre otros. Para facilitar la organización y disposición del espacio y los materiales, las cabinas cuentan con una mesa en la que estos elementos descansan. Esta mesa debe ser lo suficientemente espaciosa como para que el intérprete pueda depositar el material que necesite consultar o donde tome las notas junto con la consola, el micrófono y los auriculares. Encima de esta mesa suele haber también una lámpara o un pequeño flexo para iluminar los documentos. Debajo de ella, y a veces ligeramente ocultos tras un panel, se sitúan los cables que conectan los distintos dispositivos que he mencionado.

También es imprescindible que dentro de la cabina haya un asiento. Si está diseñada para que trabajen más intérpretes, como suele ser el caso, deberá disponer del mismo número de consolas, flexos, auriculares, asientos y micrófonos que de personas que vayan a trabajar en ella.

\section{a.3.) El sistema de sonido}

El sistema de sonido está compuesto por numerosos elementos técnicos, entre los que destacan: los micrófonos, los auriculares, los

\footnotetext{
17 Éste es el término técnico que se utiliza para referirse al cristal frontal de la cabina. El Diccionario de la Real Academia (vigésima segunda edición) recoge una acepción que avala su uso, aunque no se refiera específicamente a los cristales de las cabinas de interpretación: "bastidor con vidrios con que se cierran puertas y ventanas". Por este motivo, de aquí en adelante utilizaré siempre este vocablo.
} 
receptores $^{18}$, la unidad principal o cabina de control de sonido, transmisor, los amplificadores, los controles de nivel y los bornes de salida, el mezclador o mesa de mezclas (mixer), codificador-decodificador (CODEC), procesador digital, ecualizador, (en algunas ocasiones sistemas de grabación de sonido incorporados) etc. No obstante, el propósito de este trabajo no es el estudio de cada uno de esos elementos, sino el de ofrecer una visión más o menos general de los principales, ya que en general este sistema de sonido funciona como cualquier otro elaborado para otros propósitos. Así, por ejemplo, los auriculares o altavoces, los micrófonos y los amplificadores están presentes en todos los sistemas y funcionan de la misma manera, es decir, transmitiendo el sonido, convirtiendo el sonido en señal electrónica y aumentando el volumen de las señales eléctricas emitidas $^{19}$.

Para asegurar el buen funcionamiento del equipo y para solucionar los posibles problemas que pudieran surgir con respecto al sistema de sonido, cuando se utilicen las instalaciones de interpretación simultánea se debe contar con la presencia de un técnico.

En el caso de la interpretación simultánea, resulta imprescindible disponer de micrófonos para los oradores y para el intérprete. En caso de que se hagan turnos de preguntas y respuestas o de que el público participe por algún motivo, lo ideal es contar con un micrófono de mano que se pueda utilizar por la sala y esté conectado a la red.

Igualmente, resulta necesario instalar dispositivos para el uso de auriculares en todos los puestos: el de los intérpretes, los asistentes y los oradores (ya que en muchas ocasiones se necesita hacer interpretaciones directas e inversas). Los auriculares se conectan a los receptores mediante una clavija, igual que ocurre en otros aparatos electrónicos, como los mp3 o Ipods, por ejemplo.

Existen varios tipos de receptores: los instalados de manera fija y los portátiles. Los primeros son los puestos que pueden encontrarse en los asientos del auditorio y los portátiles los que necesitan de un bastidor o maletín para cargarse y permiten ser transportados.

El receptor del intérprete es la propia consola de interpretación. El resto de receptores sólo disponen de la función de recepción de las diferentes señales o canales y no están dotados de funciones de habla. No obstante, tienen varios pulsadores. Uno es el selector de canal, es decir, el control mediante el cual podremos seleccionar la cabina (o idioma) con la que queremos conectar. Para hacerlo sólo tendremos que mover la rueda

\footnotetext{
${ }^{18}$ En este contexto algunas empresas llaman a los receptores "delegate units". En realidad se trata de selectores de canal para el público.

${ }^{19}$ Los auriculares no suelen utilizarse en eventos donde no hay interpretación simultánea, pues con los altavoces resulta suficiente.
} 
giratoria o pulsar el botón hasta encontrar el número que deseemos. Por otro lado, también disponen de un botón regulador de volumen.

En algunos casos estos receptores pueden utilizar un sistema de infrarrojos para hacer la conexión. El sistema de infrarrojos es, tecnológicamente hablando, el más avanzado de todos, ya que proporciona una amplia cobertura con el mínimo uso de cables. La transmisión de la señal de audio de los intérpretes se realiza en la actualidad mediante potentes radiadores de infrarrojos que se ubican estratégicamente de manera que se cubra la sala. Así, se evita el tendido de la antena, no se producen interferencias, se asegura una mayor nitidez del sonido y se evita que la señal sea captada fuera de la sala, es decir, se asegura la confidencialidad. En los sistemas menos sofisticados la conexión se hace a través de cables integrados.

Para que todos estos elementos funcionen se necesita una unidad principal o cabina de control de sonido (en los equipos modernos suele ser un ordenador dotado de un software especial), que es la encargada de transmitir todas las señales y canales. De esta forma, la interpretación se transmite desde el canal de salida de la consola del intérprete hasta esta unidad, donde el sonido se procesa y se emite, después de amplificarse, hacia los receptores. Muchas veces el amplificador se encuentra integrado en la unidad principal.

El sonido digital es el de mayor calidad, ya que la información se transporta "bits" y el proceso de amplificación y separación de la señal acústica resulta más sencillo. Así, toda la señal llega a la cabina con un nivel de ruido nulo, tanto si lo hace desde otra cabina (relé) como si el sonido llega directamente desde los micrófonos de los oradores. En los sistemas modernos, la unidad de control central funciona como un ordenador, con lo que un solo técnico puede controlar una sala con varias cabinas y varios micrófonos. Si se dispone de un monitor, el operador puede controlar el funcionamiento del sistema y puede corregir fallos sin necesidad de escuchar con un receptor ${ }^{20}$.

\section{b) Otros equipos de interpretación}

En este apartado se engloban los equipos de interpretación que se utilizan en contextos diferentes a la interpretación de conferencias ${ }^{21}$. En este

\footnotetext{
${ }^{20}$ Se debe tener en cuenta que los técnicos no tienen por qué conocer las lenguas de trabajo y por lo tanto les puede resultar difícil distinguir el canal correcto entre idiomas desconocidos.

${ }^{21}$ Dichos contextos pueden ser varios, por ejemplo: la interpretación simultánea para los medios de comunicación, llamada también interpretación mediática (León, M., 2000: 270), en la que se incluyen las interpretaciones en la radio, televisión y cine; la interpretación en contextos judiciales (tribunales, juicios, etc.), las visitas a plantas, fábricas o instalaciones de negocios o turísticas, etc.
} 
caso no resulta necesario distinguir entre componentes principales y componentes secundarios, pues el diseño de los elementos de estos sistemas está pensado para ser lo más práctico y ergonómico posible y, por ende, para tener el menor número de componentes.

La diferencia primordial entre los equipos que se incluyen en este apartado y los que componen el anterior radica en el uso de la cabina. Los preceptos básicos de los receptores y el sistema de sonido descritos anteriormente son aplicables también a estos equipos.

La Asociación Internacional de Intérpretes de Conferencias denomina a los equipos para la interpretación simultánea sin cabina "bidule". No obstante, este término se refiere únicamente a lo que en este trabajo denomino "sistemas movibles", pues existe otro tipo de equipo que no precisa el uso de cabina pero que no se encuadra dentro de la definición citada más arriba. El Consejo de la Asociación considera que la interpretación simultánea sin cabina puede practicarse en los siguientes casos $^{22}$ :

- Circonstances exceptionnelles (lieu très éloigné, réunion ambulante, etc.) (cf. Art. 7, al. d du code)

- Réunions de courte durée (par exemple 2 heures)

- Nombre limité de participants (par exemple une dizaine)

- Avec des micros pour les délégués et transmission par câble vers les interprètes

- Avec des casques pour les interprètes

- Dans le respect de la norme CEI 914.

Además, dado que se trata de interpretación simultánea, deben aplicarse todas las disposiciones relativas que se detallan en el código que la misma Asociación ha creado para tales efectos. Dicho esto, voy a mencionar dos tipos diferentes de equipos de interpretación que se utilizan en contextos distintos a la interpretación de conferencias:

\section{b.1.) Sistemas portátiles movibles}

Un ejemplo claro de esta clase de equipos son los guidetour, bodypacks o infoport. Su función primordial es que el orador, el intérprete y el

\footnotetext{
${ }^{22}$ En realidad en el artículo se aboga por la supresión de esta modalidad y se hace hincapié en su uso únicamente en situaciones en las que la interpretación no se pueda efectuar de otra manera, pues según se comenta a continuación, la simultánea sin cabina no cumple con los requisitos que deben darse para que la disciplina se practique de forma eficiente: "Simultaneous interpretation without a booth (bidule) is a practice to be avoided because of the inherent difficulty - even at best - in producing the requisite high quality of interpretation. In exceptional cases, where such practice is unavoidable, ALL the criteria laid down must be met" (http://www.aiic.net/ViewPage.cfm/page634.htm).
} 
público puedan moverse por un espacio grande mientras la interpretación se lleva a cabo. Esto ocurre, por ejemplo, en las visitas guiadas (a museos o instalaciones industriales), reuniones privadas, auditorías, juntas de trabajo, cuando resulta necesario capacitar zonas especiales (como pueden ser lugares ruidosos: plataformas petrolíferas o fábricas con maquinaria), etc. Con estos sistemas, el intérprete reproduce las palabras del orador a través de un micrófono inalámbrico y el público puede seguirlas mediante el uso de unos auriculares conectados a unos receptores también inalámbricos. Estos pueden distribuirse y recogerse rápidamente.

Los sistemas movibles son muy adecuados para las interpretaciones dirigidas a grupos pequeños. Así, suelen emplearse en situaciones en las que el público es poco numeroso; de hecho, se recomienda su uso para eventos que cuenten con unas cincuenta personas máximo, ya que los receptores deben mantenerse a una estrecha distancia (10-20 metros) del intérprete para que no se pierda la recepción de la señal ${ }^{23}$.

Los componentes de estos equipos, en concreto los receptores, micrófonos y auriculares, tienen la misma forma y diseño que algunos de los que se usan en la I.C., por lo tanto, deben cumplir los mismos requisitos que los descritos anteriormente. La diferencia radica en que se suelen utilizar en lugares donde hay bastante ruido y el intérprete debe susurrar o hablar en voz baja. Los dispositivos de recepción suelen enclavarse en una pequeña carcasa portátil que también funciona como cargador de batería para los receptores sin cables.

Su ventaja, aparte de la movilidad, es que no se necesita la presencia de un técnico para que funcionen correctamente, pues no hay instalación previa ni cabinas. También resulta provechoso este sistema cuando la sala donde se va a celebrar el acto es muy pequeña y no se puede montar una cabina de interpretación o cuando se necesita interpretación para muy pocas personas. En estos casos los intérpretes se sitúan en cualquier lugar del salón donde haya visibilidad y buenas condiciones de escucha. Quizás una de las desventajas que presenta este tipo de equipos radica en que su funcionamiento se realiza por radiofrecuencia, de ahí la baja calidad de sonido.

\section{b.2.) Sistemas de interpretación telefónica}

Estos sistemas, al contrario que los anteriores, forman parte de los equipos fijos, pero también se utilizan en contextos diferentes a la interpretación de conferencias. En realidad, la modalidad de interpretación telefónica se enmarca dentro de las "interpretaciones remotas o a distancia",

\footnotetext{
${ }^{23}$ El equipo denominado infoport es un sistema de radiofrecuencia que opera en banda de 30 $50 \mathrm{MHz}$.
} 
que son la última tendencia en interpretación simultánea (sobre todo en los servicios sociales). Como equipo se emplea, tal y como su nombre indica, un teléfono para poner en contacto al intérprete con sus clientes. Se trata de un servicio que se oferta sobre todo en situaciones judiciales, hospitalarias y turísticas, aunque no está aún muy extendido en España. En cambio, en otros lugares del mundo sí está plenamente establecido. Esto ocurre, por ejemplo, en Estados Unidos, donde se puede contactar con empresas que disponen de conexiones con intérpretes que están activas las veinticuatro horas. Este servicio puede considerarse una asistencia lingüística telefónica permanente. La primera empresa que lo puso en marcha fue $A T \& T$ Language line ${ }^{24}$.

Como ocurre en otros contextos, este servicio se puede realizar en las dos modalidades de interpretación: consecutiva o simultánea. En el caso de que se elija la primera, se pueden utilizar líneas telefónicas normales; sin embargo, para efectuar la simultánea, es necesario contar con otros dispositivos más sofisticados, ya que las líneas telefónicas convencionales no separan las señales de entrada y de salida. Aunque se emplean como menos frecuencia, en la actualidad, existen unos equipos para la interpretación telefónica que incorporan sistemas de reconocimiento de voz y traducción asistida por ordenador:

...telephone-based spoken language technology is the interpretation of telephony in which two callers speaking different languages can engage in a dialogue mediated by a spoken language translation system. Such systems are currently designed to incorporate speech recognition, machine translation, and speech synthesis subsystems, and to interpret one sentence at a time. (Cohen y Ovviat, 1999: 9922.)

En el ámbito europeo son aún menos frecuentes, pero también existe un proyecto que guarda ciertas similitudes con este sistema: el llamado SUNDIAL ${ }^{25}$. Su objetivo es promover el desarrollo de la interpretación telefónica y permitir el acceso a bases de datos en diferentes idiomas. Para ello se ha creado un sistema de ordenador integrado que actúa en tiempo real y es capaz de mantener un diálogo con usuarios mediante la utilización

\footnotetext{
${ }^{24}$ Según consta en la página de la empresa, la idea partió de un joven policía que en enero de 1981 patrullaba por el barrio de San José (California) y se encontró con una situación de urgencia en la que un hombre agitaba violentamente los brazos y gritaba en una lengua desconocida para el oficial. Un año después, este policía y un amigo estudiante del Defense Language Institute de Monterrey crearon la línea lingüística. A pesar de que resulta difícil comprobar la veracidad de la historia (que probablemente esté decorada con elementos sensacionalistas para lograr fines publicitarios), lo cierto es que la idea debió fraguarse a partir de una situación en la que se produjeran serios problemas lingüísticos.

${ }^{25}$ Siglas de Speech Understanding Dialogue. Se trata de un equipo parcialmente financiado por la Comisión Europea dentro del programa ESPRIT II, proyecto P2218.
} 
de líneas de teléfono estándar. Hasta ahora se ha desarrollado en cuatro lenguas (inglés, francés, alemán e italiano) y las tareas que realiza son esencialmente la reserva de vuelos y la respuesta a preguntas sobre transporte aéreo o de trenes. Cada sistema está compuesto por cinco componentes:

A speech recognition component inputs acoustic speech signals from the telephone and outputs a word lattice; a parser extracts a plausible string from the lattice and assigns it syntactic and semantic representations; a dialogue manager gives these representations an interpretation, decides how the dialogue may continue and, if it is the system's turn to speak, plans a system utterance; a text generator constructs a detailed linguistic representation from the plan; and a textto- speech system synthesizes the representation for transmission by telephone. (McGlashan, 1992: 245).

Sin embargo, este equipo presenta dos problemas esenciales: por un lado, se necesitaría que la gestión de los diálogos fuera genérica, es decir, que el módulo de gestión pudiera generar e interpretar (en el sentido de comprender, no de traducir) los sonidos en más de una lengua y en más de un tipo de tarea. Por otro, falla la interacción con el usuario, pues en algunas ocasiones la comunicación no es fluida o el sistema de reconocimiento de voz interpreta de forma errónea el input: "for example, the user said 1 want to fly to Lannion, but the system understands London rather than Lannion" (McGlashan, 1992: 245).

Por estos y otros motivos, estos equipos para la interpretación simultánea no son aceptados por todos los expertos del gremio y hay quienes los consideran degradantes, imprecisos o de baja calidad. De hecho, se han realizado diversas investigaciones para analizar los efectos causados por este tipo de interpretación en la salud de los intérpretes y se han comparado con los que provoca una interpretación en el ámbito de conferencias. Dichos estudios ${ }^{26}$ demuestran a grandes rasgos que el nivel de estrés es algo mayor y la fatiga es muy superior.

\section{CONCLUSIONES}

La interpretación simultánea se basa en la traducción que se efectúa, de forma oral e inmediata, del discurso que el orador pronuncia en un idioma dado a otro. Partiendo de esta premisa, queda claro que la utilización de un equipo de interpretación simultánea es, si no imprescindible, muy

${ }^{26}$ Destacan, por ejemplo, los experimentos llevados a cabo por la ETI-ITU en abril de 1999, la ONU en febrero de 1999 y abril de 2001; el SCIC en enero de 2000; el Parlamento Europeo en enero de 2001 y diciembre de 2001 o el Consejo de la UE en abril de 2001, entre otros (Mouzoukaris, P., 2003: en línea). 
conveniente para el correcto ejercicio de la profesión. De esta manera, los expertos del campo de la interpretación (y también del resto de disciplinas que en ella confluyen) han de ser conscientes de la necesidad de disponer de un equipo adecuado a la situación interpretativa que cumpla con los requisitos pertinentes para facilitar la comunicación interlingüe.

Cabe destacar, que el equipo de interpretación simultánea en sí continúa basándose en los principios de los que disponían los primeros sistemas: los dos equipos iniciales funcionaban con un entramado de cables, micrófonos y auriculares conectados entre sí a través de una unidad principal, y una cabina; es decir, contaban con elementos básicos que hoy en día siguen siendo necesarios: la consola, las cabinas y el sistema de sonido. Por consiguiente, se puede concluir que el equipo de interpretación simultánea no ha experimentado una verdadera evolución, ya que las mejoras de las que goza no pueden ser consideradas como inherentes a la interpretación simultánea, aunque por supuesto, han sido y siguen siendo de gran importancia para el perfeccionamiento de la modalidad.

Quizás uno de los mayores problemas a los que el intérprete se enfrenta no es la lentitud con la que el equipo de interpretación simultánea ha evolucionado, ni que las mejoras realizadas no sean exclusivas para la interpretación; sino que más bien la dificultad radica en que, desgraciadamente, aún hoy son pocas las personas conscientes de la necesidad de trabajar utilizando un sistema de calidad. Por desgracia, con demasiada frecuencia algunas empresas encargadas del diseño de las cabinas o de la contratación de equipos portátiles no tienen en cuenta factores básicos para su correcto funcionamiento, la mayoría de las veces por desconocimiento de la profesión, y éste es un aspecto que, a mi juicio, debe cambiar cuanto antes. Esta falta de conocimiento sobre la interpretación se hace patente de forma más acuciada dentro de lo que se podrían denominar los "mercados locales", donde muchas veces podremos encontrarnos con cabinas en las que no pueden trabajar dos personas (o lo hacen incómodamente) o bien se precisa que el intérprete hable "bajito" porque no existe insonorización. De este tipo de situaciones se han hecho eco algunas organizaciones formadas por intérpretes, y es que, sin duda, uno de los logros principales que se han conseguido en la interpretación simultánea con respecto a la evolución del equipo es la creación de las asociaciones internacionales que establecen las regulaciones y requisitos con los que debe contar y los materiales necesarios para efectuar la interpretación.

Tras haber clasificado los equipos según su función e instalación y después de estudiar minuciosamente los elementos que los componen, se puede observar que es imprescindible que el intérprete pueda disponer de una instalación actual formada por unos componentes primarios que 
cumplan los requisitos mínimos (y con más o menos componentes secundarios) para poder realizar una labor de calidad. Es posible que las asociaciones y el reconocimiento de la profesión ayuden en este campo y en el futuro podamos contar con equipos especialmente diseñados para cada contexto de interpretación simultánea, ya que si existen nuevas demandas tendrá que existir también un material y un equipo adecuado y adaptado a esas nuevas condiciones de trabajo.

\section{REFERENCIAS BIBLIOGRÁFICAS}

ÁlVAREZ DE BARR, O. (1998). "La tercera pata de la interpretación de conferencias: El equipamiento técnico (primera parte)", Molinos de Viento, Revista de la Asociación de Traductores Públicos e Intérpretes de la Provincia de Buenos Aires (ATIBA). Buenos Aires. Disponible en línea en: $<$ http://www.adica.org.ar/adica/publicaciones/molinos2/body molinos2 .html> [Fecha de consulta: 20 de julio de 2010].

ASOCIACIÓN INTERNACIONAL DE INTÉRPRETES DE CONFERENCIAS (AIIC). Disponible en línea: <http://www.aiic.net/> [Fecha de consulta: 20 de agosto de 2010].

AT\&T LANGUAGE LINE SERVICES. Disponible en línea: $<$ http://www.languageline.com/page/welcome/> [Fecha de consulta: 20 de agosto de 2010].

BAIGORRI JALÓN, J. La Interpretación de conferencias: El nacimiento de una profesión. De París a Nuremberg. Granada: Comares, 2000.

J. Interpreters at the United Nations: A history. Ediciones Universidad de Salamanca, 2004.

BORDONS, B. y JIMÉNEZ, A. "La enseñanza de la interpretación" en Hurtado, A. (ed.), La Enseñanza de la Traducción, Castelló de la Plana: Universitat Jaume I, 1996.

COHEN, O. y OVVIAT, S. (1995). "The role of voice input for human-machine communication", PNAS, Vol. 92, №. 22, pp. 9921-9927. Disponible en línea en <http://www.pnas.org/content/92/22/9921.full.pdf+html> [Fecha de consulta: 20 junio 2010].

DicCiONARIO DE LA REAL ACADEMIA ESPAÑOLA. Vigésima Segunda Edición. Disponible en línea en <http://www.rae.es $>$ [Fecha de consulta: 22 de junio 2010

DRALLNY, I. La formación del intérprete de conferencias. Escuela Superior de Lenguas. Córdoba (Argentina): Ediciones El Copista, 2000.

GalBA, F. The Origins of Simultaneous Interpretation: The Nuremberg Trial, University of Ottawa Press, 1998.

García Beyaert, S. y SerRano Pons, J. "Recursos para superar las barreras lingüístico-culturales en los servicios de salud". En: 
J. Morera, A. Alonso y $\mathrm{H}$. Huerga (eds.): Manual de atención al inmigrante. Madrid / Barcelona: ERGON, 2009, pp. 53-67.

INTERNATIONAL STANDARIZATION ORGANIZATION. Cabinas de Interpretación Simultánea. Norma Internacional ISO 2603.

- Acústica. Evaluación del aislamiento acústico en los edificios y de los elementos de construcción. Norma Internacional ISO 717-1:1996. Parte 1. Aislamiento a ruido aéreo.

Cabinas de interpretación simultánea portátiles. Características generales y equipo. ISO Norma Internacional 4043:1998.

- Acústica. Absorbentes acústicos para su utilización en edificios. Evaluación de la absorción acústica. Norma Internacional ISO 11654:1997.

JORGE FIGUEROA, N. "Lengua de Signos Española: Métodos de Transcripción y Análisis Gramatical (I)", Interlingüística (Asociación de Jóvenes Lingüistas), n.ำ 9, 1998, pp. 145-150.

KEISER, W. "Selection and Training of Conference Interpreters" En Gerver, D. y Sinaico H.W. (eds.) Language Interpretation and Communication. Conference: NATO Symposium on Language Interpretation and Communication. Plenum Press [for the] NATO Scientific Affairs Division: New York / London, 1978, pp. 11-24.

, "L'Interprétation de conférence en tant que profession et les précurseurs de l'Association Internationale des Interprètes de Conférence (AIIC) 1918-1953", L'Histoire de la traduction et la traduction de l'histoire, en Meta, septiembre, vol. 49, №. 3, 2004.

LEÓN, M. Manual de Interpretación y Traducción, Madrid: Luna Publicaciones S.L., 2000.

MANUEL JEREZ, J. (coord.). Nuevas tecnologías y formación de intérpretes, Granada: Editorial Atrio, 2003.

MARTIN, A. "La interpretación de conferencia en el mercado libre" En Kelly, D., (ed.), La traducción y la interpretación en España hoy: perspectivas profesionales. Granada: Comares, 2000, pp. 171-188.

MCGLASHAN, N. et alii. "Dialogue management for telephone information system", Proceedings of the International Conference on Applied Language Processing, 1992 Disponible en línea en $<$ http://acl.Idc.upenn.edu/A/A92/A92-1040.pdf> [Fecha de consulta: 20 de junio de 2010].

MOUZOURAKIS, P.T. "That feeling of being there: vision and presence in remote interpreting", AllC webmagazine. Vol. 3, 2003. Disponible en línea en <http://www.aiic.net/ViewPage.cfm?page $\mathrm{id}=1173>$ [Fecha de consulta: 9 de julio de 2011]. 
MUNDAY, J. Introducing Translation Studies: Theories and Applications. Routledge: New York / London, 2001.

WADENSJÖ, C. "Community Interpreting" En: Routledge encyclopedia of translation studies de Baker, M. (ed.), Routledge: New York / London, 1998. 